\title{
The ruptured Achilles tendon: operative and non-operative treatment options
}

\author{
Joshua A. Metzl · Christopher S. Ahmad • \\ William N. Levine
}

Published online: 20 March 2008

(C) The Author(s) 2008

\begin{abstract}
The Achilles tendon is the strongest and thickest tendon in the human body. Like any other tendon in the body, however, it is susceptible to rupture. Many surgeons advocate early operative repair of the ruptured Achilles tendon, citing decreased re-rupture rates and improved functional outcome. Waiting for surgical repair for longer than one month may lead to inferior functional results postoperatively. Non-operative treatment has higher re-rupture rates as compared to surgically repaired tendons, but may be the treatment of choice in some patients. While for many years, patients were rigidly immobilized in a nonweightbearing cast for 6-8 weeks postoperatively, newer studies have shown excellent results with early weightbearing, and this is quickly becoming the standard of care amongst many physicians.
\end{abstract}

Keyword Achilles tendon

\section{Introduction}

The Achilles tendon is the thickest and strongest tendon in the human body. Like any other tendon in the body, however, it is susceptible to overuse and rupture (Fig. 1a and b). Achilles tendon ruptures are increasing in frequency due to an overall increase in athletic activity, especially in men in their thirties and forties. About 18 out of 100,000 humans rupture their Achilles tendon each year,

\footnotetext{
J. A. Metzl · C. S. Ahmad · W. N. Levine ( $\square)$

Department of Orthopedic Surgery, Center for Shoulder, Elbow and Sports Medicine, Columbia University Medical Center, 622 West 168th Street, PH1117, New York, NY 10032, USA

e-mail:WNL1@columbia.edu
}

depending on the social environment [1]. Acute ruptures of the Achilles tendon occur most often in men in the third and fourth decades of life who intermittently participate in athletic activity.

The spectrum of Achilles tendon ruptures includes both acute and chronic injuries. Treatment options include operative repair with postoperative immobilization, operative repair with accelerated rehabilitation using early weight bearing, as well as non-operative treatment. Clinical evaluations of non-operative treatment have demonstrated a re-rupture rate of 10-30\% [2]. Other drawbacks of nonoperative treatment include decreased plantar flexion and decreased endurance when compared to surgically repaired tendons [3]. Some reports, however, suggest that the results of non-operative management are equivalent to operative repair [4-6].

Several current studies favor operative repair via open or percutaneous techniques in younger, active patients who wish to return to pre-injury activities [7, 8]. In addition, rerupture is less in surgically repaired Achilles tendons [9]. Complications of operative repair include infection and poor wound healing. Ultimately, decision-making regarding the treatment options relies on many factors including patient age, chronicity of the tear, functional demand, medical comorbidities, and patient expectations.

\section{Anatomy}

The Achilles tendon is composed of tendinous fibers contributed by the gastrocnemius and soleus muscles. These fibers then coalesce and insert on the calcaneal tuberosity. The blood supply to the Achilles tendon has three principle sources: the musculotendinous junction, the osseous insertion, and multiple vessels in the mesotenon [10]. Injection 

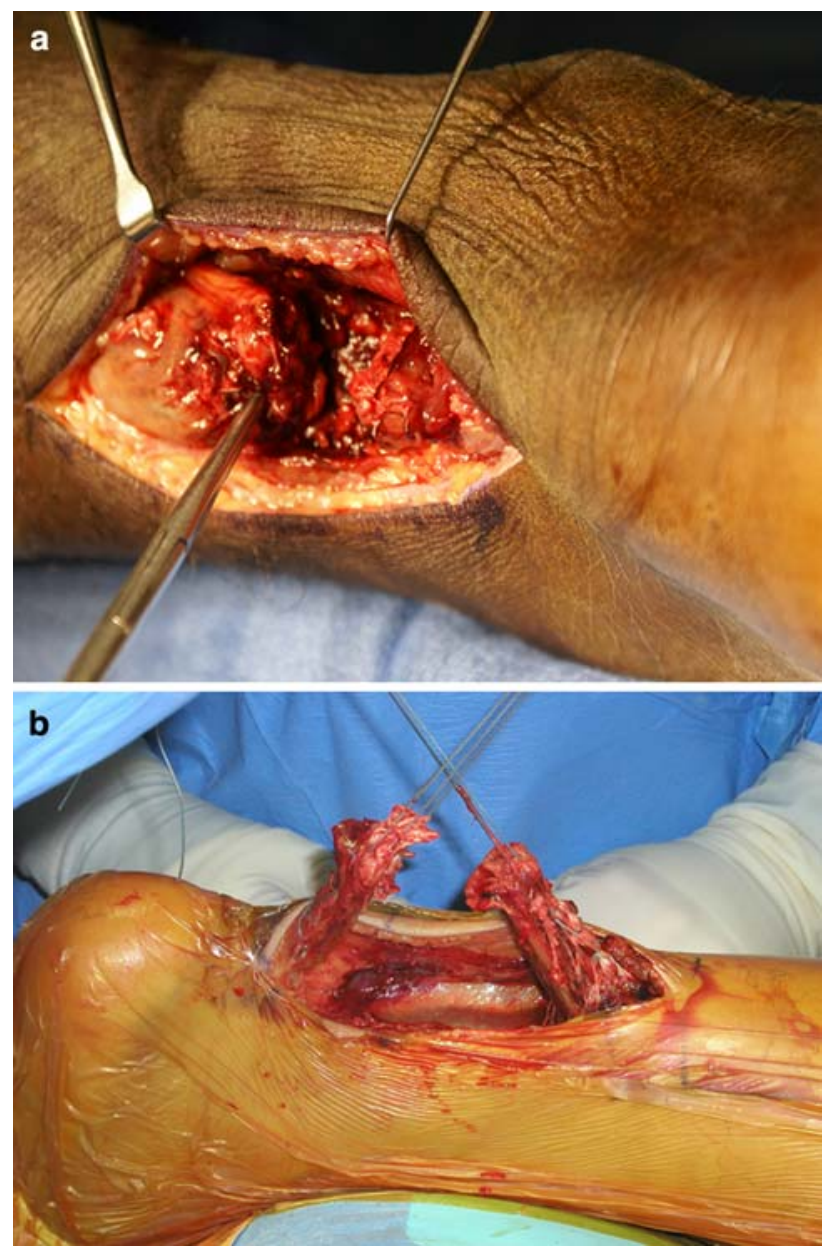

Fig. 1 (a) and (b) - Intra-operative view of ruptured Achilles tendon

studies using computer assisted imaging show a reduction in the number and the mean relative area of mesotenal vessels in the mid-section of the tendon. Similarly, the number of intratendinous vessels is fewest $4 \mathrm{~cm}$ from the calcaneal insertion [11].

\section{Physical examination}

Diagnosis of an acute Achilles tendon rupture can usually be made by history and physical examination alone. MRI or ultrasound can be helpful in cases of suspected rupture for pre-operative planning, but is usually unnecessary. The patient will often describe abrupt severe pain over the tendon with an activity requiring forceful plantar flexion such as while playing tennis. Often the patient will feel a "pop" or have the sensation of being hit in the back of the heel. Physical examination signs that suggest Achilles tendon rupture include weakness of plantar flexion and a palpable defect over the posterior calf. A Thompson test is performed by squeezing the calf and observing motion at

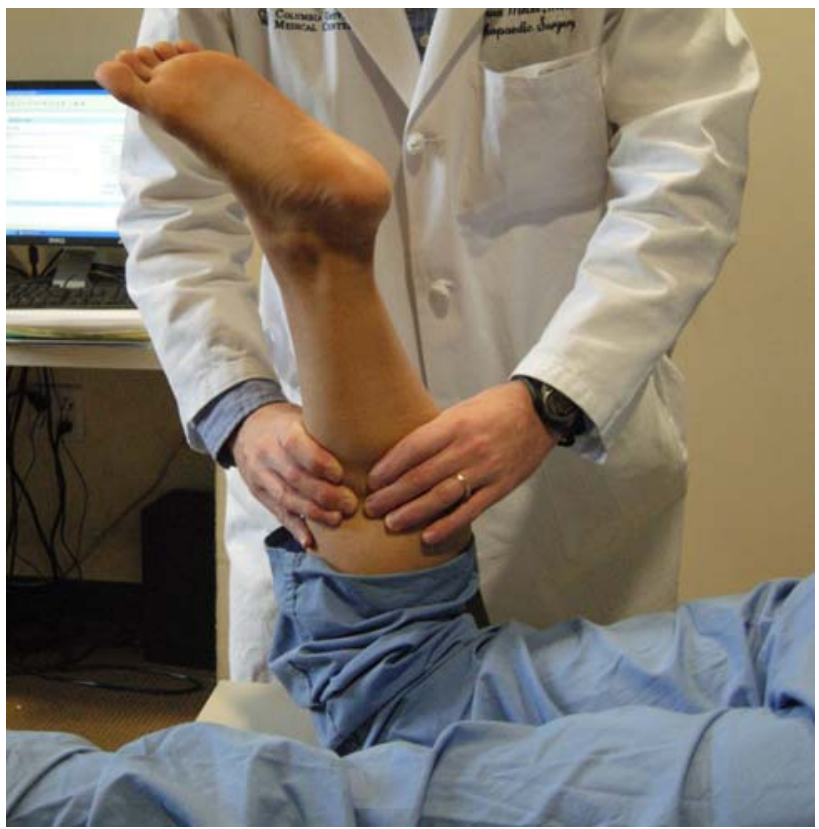

Fig. 2 Normal Thompson's test. Note plantar flexion of foot with squeezing of the calf. Indicative of intact Achilles tendon

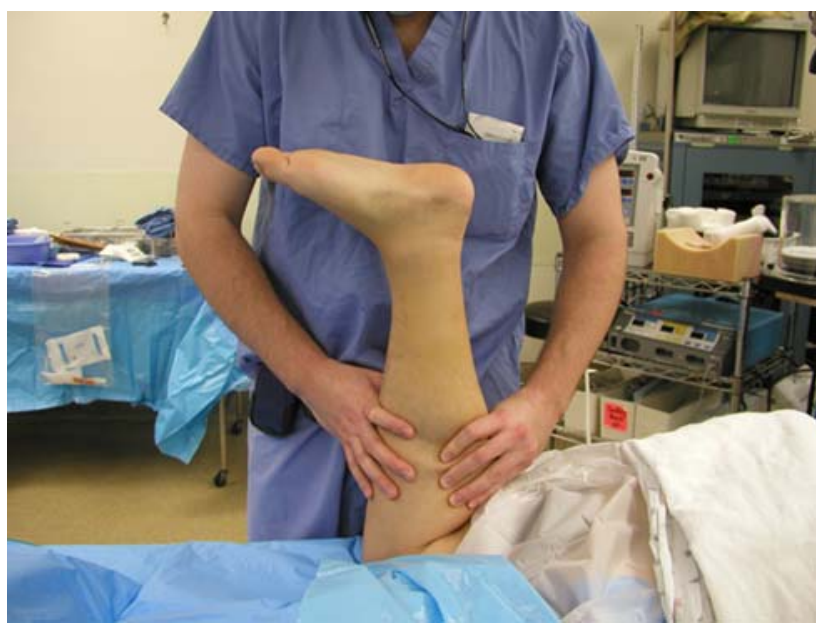

Fig. 3 Thompson's test in patient with Achilles tendon rupture. Note lack of plantar flexion with squeezing of the calf demonstrating a ruptured Achilles tendon

the foot. With an intact tendon, the foot planterflexes with squeezing the calf (Fig. 2). With a rupture, plantar flexion does not occur with squeezing the calf [12] (Fig. 3).

\section{Operative versus non-operative management}

Controversy regarding operative versus non-operative treatment focuses on re-rupture rates and wound complications related to surgery. Khan et al. compared the results of all randomized trials comparing surgical and conservative management in a meta-analysis. Results gave a pooled 
re-rupture rate of $3.5 \%$ in the operatively treated group and $12.6 \%$ in the non-operative group. Complications such as adhesions, altered sensation, and wound infection, however, were more common in operatively treated patients with $34.1 \%$ in the operative group versus $2.1 \%$ in the nonoperative group [6, 13, 14].

In a large prospective study of 196 consecutive patients with acute Achilles tendon ruptures that were treated with 8 weeks of casting and immediate postoperative weightbearing, the re-rupture frequency was 7\% [15]. This is only slightly higher than most results in operatively treated groups $[2-4,6,15]$. Due to the similar percentage of rerupture between most studies advocating surgery and their promising results with non-operative management, Ingvar et al. question the necessity for surgical repair of Achilles tendons, with all of its risks and high cost [15].

\section{Open versus percutaneous repair}

In 1977, Ma and Griffith introduced percutaneous Achilles tendon repair. This technique involves passing a suture through small stab wounds above and below the tendon tear. The original article reported on 18 patients with only two minor complications [16]. Subsequent groups have reported similar results. Cretnik et al. prospectively studied 134 percutaneous repairs and found a rerupture rate and return to pre injury activities comparable to open procedures. Complications included complete and partial reruptures, sural neuritis, and superficial wound infection [17]. Percutaneous repair has the added benefit of being done under local anesthesia without a tourniquet.

Lim et al. showed no significant difference between open compared to percutaneously repaired tendons, but a significantly higher rate of wound infection in the open group compared to the percutaneous group [18].

\section{Postoperative management}

Postoperative management is also controversial. Historically, patients were immobilized in a rigid cast for at least 4-6 weeks to allow for presumed tendon healing. Current trends, however, favor minimizing postoperative immobilization and focus on early weightbearing with excellent results. In a rat model of tendon rupture using wheel running for early tendon motion, Bring et al. were able to show that physical activity speeds up tendon healing as compared to plaster immobilization. This suggests that that there may be some stimulatory effect of mechanical loading on tendon healing [19]. In a clinical prospective study with full postoperative weight bearing in a controlled ankle motion walker accompanied by active stretching, Jacob and Paterson showed few minor complications, high patient satisfaction, and no re-ruptures. Patients with both acute and chronic tears repaired with an open technique did equally well under this treatment protocol [20]. In their meta-analysis of randomized trials comparing early postoperative weightbearing with cast immobilization, Suchak et al. found no difference in re-rupture rates and more excellent rated subjective patient responses. Patients preferred the early function protocol with a mobile cast for multiple reasons including a decrease in leg edema, the ability to bear weight, and easier and faster ability to obtain normal gait after removal of the cast [21].

Impact of time until surgery

Sorrenti et al. divided their treatment groups into three sections based on the number of days between Achilles tendon rupture and surgery: acute tears ( $<7$ days), sub chronic tears ( $>7$ days but $<4$ weeks), and chronic tears ( $>4$ weeks). All patients resumed their normal activities within 100 days of operative repair, regardless of time until surgery. Thus, according to this study, there does not appear to be an advantage of early operative repair over delayed surgical treatment [22]. The main concern to prolonged treatment delay after Achilles tendon rupture is weakness of plantar flexion.

\section{Conclusion}

Surgical repair of the ruptured Achilles tendon is a controversial topic. Many authors advocate surgical repair, mostly citing decreased re-rupture rates and improved functional outcome. No studies show a clear advantage to early surgical intervention over delayed repair. Waiting for surgical repair for longer than 1 month may show worse functional results postoperatively. Non-operative treatment has shown similar re-rupture rates as compared to surgically repaired tendons. Any patient undergoing Achilles tendon repair should understand the inherent risks of an invasive procedure including bleeding, infection, and pain at the surgical site. In our practice, we offer surgical repair of the ruptured Achilles tendon for those patients who wish to return to an active lifestyle. While for many years, patients were rigidly immobilized for 6-8 weeks postoperatively, new studies have shown excellent results with early weightbearing, and this is quickly becoming the standard of care amongst many physicians.

Open Access This article is distributed under the terms of the Creative Commons Attribution Noncommercial License which permits any noncommercial use, distribution, and reproduction in any medium, provided the original author(s) and source are credited. 


\section{References}

1. Gebauer M, et al. Mechanical evaluation of different techniques for Achilles tendon repair. Arch Orthop Trauma Surg. 2007;127(9):795-9.

2. Strauss EJ, et al. Operative treatment of acute Achilles tendon ruptures: an institutional review of clinical outcomes. Injury. 2007;38(7):832-8.

3. Kocher MS, et al. Operative versus nonoperative management of acute Achilles tendon rupture: expected-value decision analysis. Am J Sports Med. 2002;30(6):783-90.

4. Cetti R, et al. Operative versus nonoperative treatment of Achilles tendon rupture. A prospective randomized study and review of the literature. Am J Sports Med. 1993;21(6):791-9.

5. McComis GP, Nawoczenski DA, DeHaven KE. Functional bracing for rupture of the Achilles tendon. Clinical results and analysis of ground-reaction forces and temporal data. J Bone Joint Surg Am. 1997;79(12):1799-808.

6. van der Linden-van der Zwaag HM, Nelissen RG, Sintenie JB. Results of surgical versus non-surgical treatment of Achilles tendon rupture. Int Orthop. 2004;28(6):370-3.

7. Lewis N, Quitkin HM. Strength analysis and comparison of the Teno fix tendon repair system with the two-strand modified Kessler repair in the Achilles tendon. Foot Ankle Int. 2003;24(11):857-60.

8. Lansdaal JR, et al. The results of 163 Achilles tendon ruptures treated by a minimally invasive surgical technique and functional aftertreatment. Injury. 2007;38(7):839-44.

9. Khan RJ, et al. Treatment of acute achilles tendon ruptures. A meta-analysis of randomized, controlled trials. J Bone Joint Surg Am. 2005;87(10):2202-10.

10. Saltzman CL, Tearse DS. Achilles tendon injuries. J Am Acad Orthop Surg. 1998;6(5):316-25.

11. Carr AJ, Norris SH. The blood supply of the calcaneal tendon. J Bone Joint Surg Br. 1989;71(1):100-1.
12. Thompson TC. A test for rupture of the tendo achillis. Acta Orthop Scand. 1962;32:461-5.

13. Xia W, et al. Spontaneous recovery of injured Achilles tendon in inducible nitric oxide synthase gene knockout mice. Inflamm Res. 2006;55(1):40-5.

14. Moller M, et al. Acute rupture of tendon Achillis. A prospective randomised study of comparison between surgical and non-surgical treatment. J Bone Joint Surg Br. 2001;83(6):843-8.

15. Ingvar J, Tagil M, Eneroth M. Nonoperative treatment of Achilles tendon rupture: 196 consecutive patients with a 7\% re-rupture rate. Acta Orthop. 2005;76(4):597-601.

16. Ma GW, Griffith TG. Percutaneous repair of acute closed ruptured achilles tendon: a new technique. Clin Orthop Relat Res. 1977(128):247-55.

17. Cretnik A, Kosanovic M, Smrkolj V. Percutaneous suturing of the ruptured Achilles tendon under local anesthesia. J Foot Ankle Surg. 2004;43(2):72-81.

18. Lim J, Dalal R, Waseem M. Percutaneous vs. open repair of the ruptured Achilles tendon-a prospective randomized controlled study. Foot Ankle Int. 2001;22(7):559-68.

19. Bring DK, et al. Physical activity modulates nerve plasticity and stimulates repair after Achilles tendon rupture. J Orthop Res. 2007;25(2):164-72.

20. Jacob KM, Paterson R. Surgical repair followed by functional rehabilitation for acute and chronic achilles tendon injuries: excellent functional results, patient satisfaction and no reruptures. ANZ J Surg. 2007;77(4):287-91.

21. Suchak AA, et al. Postoperative rehabilitation protocols for Achilles tendon ruptures: a meta-analysis. Clin Orthop Relat Res. 2006;445:216-21.

22. Sorrenti SJ. Achilles tendon rupture: effect of early mobilization in rehabilitation after surgical repair. Foot Ankle Int. 2006;27(6):407-10. 